\title{
Diversidad y composición estacional de la escorpiofauna (Arachnida: Scorpiones) del archipiélago Los Choros (Región de Coquimbo, Chile)
}

\section{Diversity and seasonal composition of the scorpiofauna (Arachnida: Scorpiones) of the Choros archipelago (Coquimbo Region, Chile)}

\author{
Jaime Pizarro-Araya ${ }^{1 *}$, Andrés A. Ojanguren-Affilastro², Francisco López-Cortés ${ }^{3}$, Pablo \\ Agusto ${ }^{1}$, Raúl Briones $^{4} \&$ Jorge Cepeda-Pizarro ${ }^{1}$ \\ ${ }^{1}$ Laboratorio de Entomología Ecológica, Departamento de Biología, Facultad de Ciencias, Universidad de La Serena, Chile. \\ 2División Aracnología, Museo Argentino de Ciencias Naturales Bernardino Rivadavia, Buenos Aires, Argentina. \\ ${ }^{3}$ Departamento de Biología, Facultad de Ciencias, Universidad de La Serena, Chile. \\ ${ }^{4}$ Bioforest S.A. División Manejo Ecosistémico. Programa Conservación de Fauna. Km 15 Concepción, Chile. \\ *E-mail: japizarro@userena.cl
}

\begin{abstract}
RESUMEN
Se estudió la composición taxonómica, la abundancia y la estructura espacial y temporal de la escorpiofauna presente en el archipiélago de Los Choros (Región de Coquimbo, Chile). Se registró la presencia de cuatro especies: Bothriurus coriaceus Pocock 1893, Brachistosternus (Brachistosternus) roigalsinai Ojanguren-Affilastro 2002, Brachistosternus (Brachistosternus) cepedai Ojanguren-Affilastro et al. 2007a (Bothriuridae) y Caraboctonus keyserlingi Pocock 1893 (Caraboctonidae). Se registraron diferencias entre las especies de escorpiones en sus niveles de abundancia total, presencia y abundancia tanto estacional como en los diferentes ambientes pedológicos. El 53,1\% del total de la abundancia correspondió a $B$. coriaceus, que estuvo presente en ocho de los nueve ambientes pedológicos analizados y mostró actividad en las tres estaciones del año muestreadas, al igual que Br. roigalsinai, el cual representó el 30,3\% de la abundancia total y fue registrado en siete ambientes pedológicos. La especie $B r$. cepedai tuvo baja abundancia (4,1\%) y su distribución estuvo restringida a los ambientes dunarios, en los meses de primavera y verano. Por otra parte, Caraboctonus keyserlingi representó la situación opuesta, con un $12,5 \%$ de la abundancia, restringida a los ambientes de la isla Choros y a los meses de primavera. No existe una estructuración temporal, pero es reconocible un patrón de estructuración espacial. En base a estos antecedentes proponemos que las diferencias en las especies, tanto en su densidad, como en las estrategias de uso y ocupación de los ambientes podrían explicar los patrones observados.
\end{abstract}

Palabras Claves: desierto costero, escorpiones, sistemas insulares, Reserva Nacional Pingüino de Humboldt, Chile.

\begin{abstract}
We studied the taxonomic composition, abundance and degree of spatial and temporal structure of the scorpion fauna present in Los Choros Archipelago (Coquimbo Region, Chile). We recorded the presence of four species: Bothriurus coriaceus Pocock 1893, Brachistosternus (Brachistosternus) roigalsinai Ojanguren-Affilastro 2002, Brachistosternus (Brachistosternus) cepedai Ojanguren-Affilastro et al. 2007a (Bothriuridae) and Caraboctonus keyserlingi Pocock 1893 (Caraboctonidae). Among scorpion species we found differences in the levels of total abundance, seasonal presence and abundance as well as in the different pedological environments. Bothriurus coriaceus accounted for $53.1 \%$ of the total abundance, it was recorded in eight of the nine pedological environments studied and showed activity in the three sampled seasons. Likewise, Br. roigalsinai, accounted for $30.3 \%$ of the total abundance and it was recorded in seven pedological environments. The species Br. cepedai had low abundance (4.1\%) and its distribution was restricted to dune environments and to spring-summer seasons. The species Caraboctonus keyserlingi accounted for $12.5 \%$ of the abundance and its distribution was spatially restricted to the Los Choros island and spring season months. There is not a temporal structure but a spatial structure pattern is recognized. Based on this background we propose that differences in species adaptability to temporal density as well as their strategies of use and environment occupation could explain the observed patterns.
\end{abstract}

KEYwords: coastal desert, scorpions, island systems, Pingüino de Humboldt National Reserve, Chile. 


\section{INTRODUCCIÓN}

El desierto costero transicional de Chile (DCT, $25^{\circ}-32^{\circ}$ S) se ubica entre el desierto hiperárido de Atacama por el norte y la zona de tipo mediterráneo por el sur, abarcando una extensión latitudinal de $\sim 400 \mathrm{~km}, \mathrm{y}$ corresponde al límite septentrional del "hotspot chileno" (Gaston 2000; Klausmeyer \& Shaw 2009), el cual representa un foco de diversidad y endemismo vegetacional (Armesto et al. 1993; Vidiella et al. 1999; Cavieres et al. 2001, 2002). En los últimos años ha aumentado el conocimiento de la fauna del DCT, en particular de los artrópodos epígeos (Pizarro-Araya \& Jerez 2004; Cepeda-Pizarro et al. 2005a, 2005b; Agusto et al. 2006; Valdivia et al. 2008; Ojanguren-Affilastro et al. 2012; Flores \& Pizarro-Araya 2012; Pizarro-Araya et al. 2012a, 2012b; Elgueta 2013; Laborda et al. 2013; Ojanguren-Affilastro \& Pizarro-Araya 2014; Bustamante et al. 2014). La mayor parte de las investigaciones se han realizado en las áreas continentales, y sólo unas pocas se han realizado en áreas insulares. En estas áreas insulares se han descrito varias especies endémicas (Pizarro-Araya \& Flores 2004; Ojanguren-Affilastro et al. 2007a; PizarroAraya et al. 2012a), se ha analizado el ensamble de tenebriónidos epígeos (Alfaro et al. 2009) y sus variaciones morfológicas (Benítez et al. 2014) y se ha comparado la fauna de Solifugae (Arachnida) entre las áreas insulares y continentales aledañas (Valdivia et al. 2011).

Entre los arácnidos, los escorpiones cumplen un rol importante en la estructura y función de los ecosistemas áridos y semiáridos (Polis 1980, 1993; Williams 1987; Prendini 2001), participando en los procesos de fragmentación biológica, en el ciclo de nutrientes, y en las cadenas tróficas locales, ejerciendo roles como depredadores y formando parte de la dieta de organismos consumidores, principalmente vertebrados (Polis 1980; Polis \& Farley 1980; Williams 1987).

En Chile, la escorpiofauna está representada por 50 especies, pertenecientes a las familias Bothriuridae, Buthidae (sólo especies introducidas) y Caraboctonidae (Mattoni 2007; Ojanguren-Affilastro \& Ramírez 2009; Ojanguren-Affilastro et al. 2010, 2011, 2012). En el DCT se han registrado 20 especies, pertenecientes a las familias Bothriuridae y Caraboctonidae; y se han documentado elevados niveles de diversidad y endemismo (Agusto et al. 2006; Ojanguren-Affilastro et al. 2007a, 2012; OjangurenAffilastro \& Pizarro-Araya 2014).

Nuestros estudios sobre la escorpiofauna del DCT, realizados hasta el momento, han estado centrados en las dunas costeras continentales ubicadas frente a la Reserva Nacional Pingüino de Humboldt (Agusto et al. 2006; Ojanguren-Affilastro et al. 2007a), área que pertenece al Sistema Nacional de Áreas
Silvestres Protegidas del Estado (SNASPE). Parte de esta reserva abarca el archipiélago de Los Choros, que incluye tres islas: Choros, Damas y Gaviota. A la fecha no se han realizado estudios descriptivos de estos artrópodos a nivel insular, ni estudios comparativos en términos de abundancia y riqueza con las comunidades continentales aledañas. Los objetivos del presente trabajo son: i) describir la diversidad taxonómica, riqueza y abundancia de escorpiones presentes en el archipiélago de Los Choros y ii) comparar la composición de la escorpiofauna entre estaciones y entre ambientes pedológicos presentes en este archipiélago.

\section{MATERIALES Y MÉTODOS}

Área de estudio

El estudio se realizó el año 2006 en el archipiélago de Los Choros, el cual incluye las islas: Choros $\left(29^{\circ} 15^{\prime} \mathrm{S}, 71^{\circ} 32^{\prime}\right.$ O), con 322 ha; Damas ( $29^{\circ} 13^{\prime} \mathrm{S}, 71^{\circ} 31^{\prime}$ O), con 56 ha, y Gaviota ( $\left.29^{\circ} 15^{\prime} \mathrm{S}, 71^{\circ} 28^{\prime} \mathrm{O}\right)$, con 182 ha (Fig. 1). Estas islas están situadas en el extremo nor-occidental de la zona de Punta de Choros, a $114 \mathrm{~km}$ al norte de La Serena (Región de Coquimbo, Chile) (Castro \& Brignardello 2005). El área corresponde a una zona desértica costera, con un clima de tendencia mediterránea (di Castri \& Hajek 1976) con presencia de neblina. La temperatura es relativamente baja para su ubicación latitudinal, y debido a la influencia oceánica de la corriente de Humboldt posee una baja amplitud térmica diaria y anual (Armesto et al. 1993). Las precipitaciones son escasas e irregulares, y se concentran en los meses de invierno (de Mayo a Agosto) con 29 días de lluvia en promedio y con montos que no superan los 90 $\mathrm{mm}$ anuales. Hay años secos con menos de $25 \mathrm{~mm}$ anuales y años húmedos con aportes de más de $175 \mathrm{~mm}$, en ciclos irregulares y aparentemente relacionados con la influencia de ENOS (El Niño-Oscilación del Sur) (Novoa \& Villaseca 1989). Las características geomorfológicas y biofísicas del área de estudio se encuentran en Castro \& Brignardello (2005) y la descripción florística en Marticorena et al. (2001) y Arancio \& Jara (2007).

Metodología de muestreo

Se seleccionaron nueve sitios (tres por isla) que representaron los ambientes pedológicos característicos, identificados como: i) Isla Choros, estepa costera (ECCh), hábitat pedregoso interior (HPICh) y hábitat pedregoso interior sur (HPISCh); ii) Isla Damas, duna costera norte (DCNDa), duna costera sur (DCSDa) y duna interior (DIDa); iii) Isla Gaviota, estepa costera (ECGa), duna costera norte (DCNGa) y duna interior (DIGa) (Tabla 1) (Fig. 1). En cada uno de los nueve sitios, se instalaron dos parcelas de 4 x 5 $\mathrm{m}$, cada una de las cuales estuvo constituida por 20 trampas de intercepción dispuestas en una grilla. Cada trampa consistió en un dispositivo formado por dos vasos plásticos 
dispuestos uno dentro del otro, con el vaso interior de fácil remoción (sensu Cepeda-Pizarro et al. 2005a, 2005b). Las dimensiones de ambos vasos fueron 7,4 y 7,6 cm de diámetro x 10,2 y 12,0 cm de alto, respectivamente. El vaso interior fue llenado, hasta los dos tercios de su capacidad, con una mezcla de agua de mar, detergente doméstico y alcohol etílico $95^{\circ}$. Las trampas operaron durante cuatro días (tres noches activas), en los meses de Agosto, Octubre y Diciembre del 2006. Las trampas de intercepción fueron complementadas con prospecciones nocturnas con luz
UV, que permite la detección de los escorpiones por la fluorescencia del tegumento (Stahnke 1972). El material colectado fue procesado y determinado a nivel específico siguiendo a Mattoni \& Acosta (2006), Ojanguren-Affilastro (2002, 2005) y a Ojanguren-Affilastro et al. (2007a, 2007b). Los especimenes capturados están depositados en la colección aracnológica del Laboratorio de Entomología Ecológica de la Universidad de La Serena (LEULS) y en el Museo Argentino de Ciencias Naturales Bernardino Rivadavia (MACN).

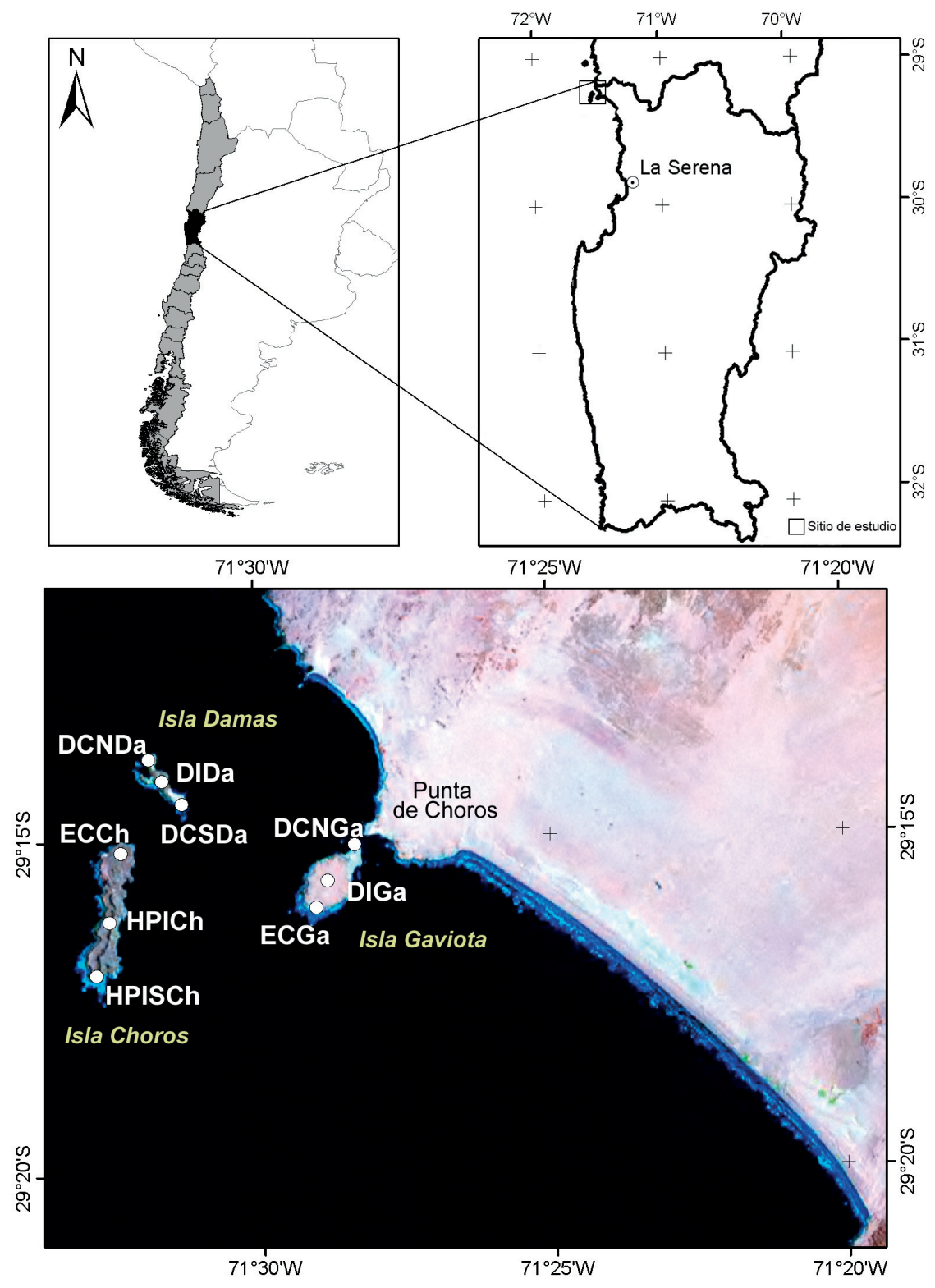

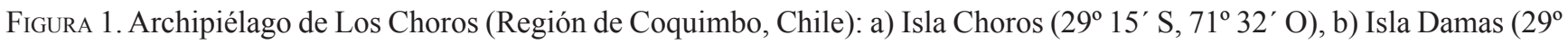
$\left.13^{\prime} \mathrm{S}, 71^{\circ} 31^{\prime} \mathrm{O}\right)$, c) Isla Gaviota (29 $\left.15^{\prime} \mathrm{S}, 71^{\circ} 28^{\prime} \mathrm{O}\right)$.

Figure 1. Los Choros Archipelago (Coquimbo Region, Chile): a) Choros Island (29 $\left.15^{\prime} \mathrm{S}, 71^{\circ} 32^{\prime} \mathrm{W}\right)$, b) Damas Island (29 $\left.13^{\prime} \mathrm{S}, 71^{\circ} 31^{\prime} \mathrm{W}\right)$, c) Gaviota Island (29 $\left.15^{\prime} \mathrm{S}, 71^{\circ} 28^{\prime} \mathrm{W}\right)$. 
Diversidad y estacionalidad de escorpiones insulares: JAIME PIZARro-AraYA ET AL.

TABLA 1. Especies de escorpiones presentes en los diferentes ambientes pedológicos en las islas del archipiélago de Los Choros.

TABLE 1. Species of scorpions present in different pedological environments in the islands of Los Choros Archipelago.

\begin{tabular}{|c|c|c|c|c|c|c|c|c|c|}
\hline \multirow[b]{3}{*}{ ESPECIE } & \multicolumn{9}{|l|}{ ISLA } \\
\hline & \multicolumn{3}{|l|}{ Choros } & \multicolumn{3}{|l|}{ DAMAS } & \multicolumn{3}{|l|}{ Gaviota } \\
\hline & $\mathrm{ECCh}$ & HPICh & HPISCh & DCNDa & DIDa & DCSDa & DCNGa & $\mathrm{DIGa}$ & ECGa \\
\hline Br. roigalsinai & + & & & + & + & + & + & + & + \\
\hline Br. cepedai & & & & & & & & + & + \\
\hline B. coriaceus & + & + & + & + & + & + & + & + & \\
\hline C. keyserlingi & + & + & + & & & & & & \\
\hline TOTAL & 3 & 2 & 2 & 2 & 2 & 2 & 2 & 3 & 2 \\
\hline \multicolumn{10}{|c|}{$\begin{array}{l}\text { Abreviaciones: Isla Choros, estepa costera (ECCh), hábitat pedregoso interior (HPICh) y hábitat pedregoso interior sur (HPISCh); Isla } \\
\text { Damas, duna costera norte (DCNDa), duna costera sur (DCSDa) y duna interior (DIDa) e Isla Gaviota, estepa costera (ECGa), duna } \\
\text { costera norte (DCNGa) y duna interior (DIGa). } \\
\text { Abbreviations: Choros Island, coastal steppe (ECCh), interior stony habitat (HPICh) and coastal stony habitat south HPISCh); Damas } \\
\text { Island, north coastal dunes (DCNDa), south coastal dunes (DCSDa) and interior dunes (DIDa); Gaviota Island, coastal steppe (ECGa), } \\
\text { north coastal dunes (DCNGa) and interior Dunes (DIGa). }\end{array}$} \\
\hline
\end{tabular}

ANÁLISIS DE DATOS

Se analizó la diversidad y abundancia de la escorpiofauna en cada isla (Isla Choros (Ch), Isla Damas (Da), e Isla Gaviota (Ga)), en tres estaciones del año: Invierno (agosto), Primavera (octubre) y Verano (diciembre). Se calcularon los siguientes estimadores: (1) riqueza o número total de especies; (2) abundancia; (3) índice de diversidad de Shannon-Weiner $\left(H^{\prime}\right)$ y (4) equidad de Pielou ( $J^{\prime}$ ) (Magurran 1988).

El grado de estructuración estacional y por ambiente pedológico se determinó usando un análisis de ordenación Cluster sobre el cálculo de la matriz de similitud, tanto de Bray-Curtis como de Jaccard (Faith et al. 1987). Tanto para evaluar la significancia estadística de las diferencias entre estaciones del año, como entre unidades, se utilizó un Análisis de Similitudes ANOSIM de una vía basado en las medidas de distancia de los índices de Bray-Curtis y de Jaccard, con apoyo del programa PAST v2.1 (Hammer et al. 2001).

\section{RESULTADOS}

DIVERSIDAD TAXONÓMICA, RIQUEZA Y ABUNDANCIA

En el sistema insular estudiado se capturaron un total de 145 especímenes que corresponden a las especies; Bothriurus coriaceus Pocock 1893, Brachistosternus roigalsinai Ojanguren-Affilastro 2002, Brachistosternus cepedai Ojanguren-Affilastro et al. 2007a (Bothriuridae) y Caraboctonus keyserlingi Pocock 1893 (Caraboctonidae). La especie Bothriurus coriaceus fue la más abundante $(53,1$ $\%$ del total de la abundancia), seguida por Brachistosternus roigalsinai (30,3\%), Caraboctonus keyserlingi (12,5\%) y Brachistosternus cepedai (4,1\%).

En las islas Choros y Gaviota se registró la mayor riqueza específica, con tres de las cuatro especies presentes en el Archipiélago (Fig. 2). La duna costera norte y la estepa costera fueron los ambientes pedológicos donde se registró la mayor riqueza específica $(\mathrm{S}=3)$ y abundancia total de individuos. Los restantes ambientes presentan una riqueza específica $(\mathrm{S}=2)$ y abundancia total menor, destacándose en este aspecto los ambientes de duna interior (DIGa) y de estepa costera (ECGa), con tres y nueve individuos, respectivamente. Bothriurus coriaceus se encuentra en ocho de los nueve ambientes pedológicos muestreados, con las mayores abundancias en los ambientes de estepa y pedregosos (Tabla 1), mientras que Br. roigalsinai se encuentra en siete ambientes, con abundancias mayores en los ambientes de dunas (costera e interior). Brachistosternus cepedai se encuentra exclusivamente en dunas de la isla Gaviota, mientras que $C$. keyserlingi se encuentra exclusivamente en isla Choros (Fig. 3).

La mayor riqueza específica y abundancia se encontró en primavera (66\% del total de capturas), seguido por el verano (22\%) y el invierno (12\%) (Fig. 4). El mayor número de especies se encontró en la isla Gaviota en primavera. La isla Choros mostró una menor riqueza de especies en invierno y la incorporación al registro de $C$. keyserlingi en primavera, con una importante contribución a la abundancia total. En la isla Damas se observó un aumento en la riqueza de escorpiones desde el invierno hasta el verano, con los mayores valores de abundancia en primavera. 


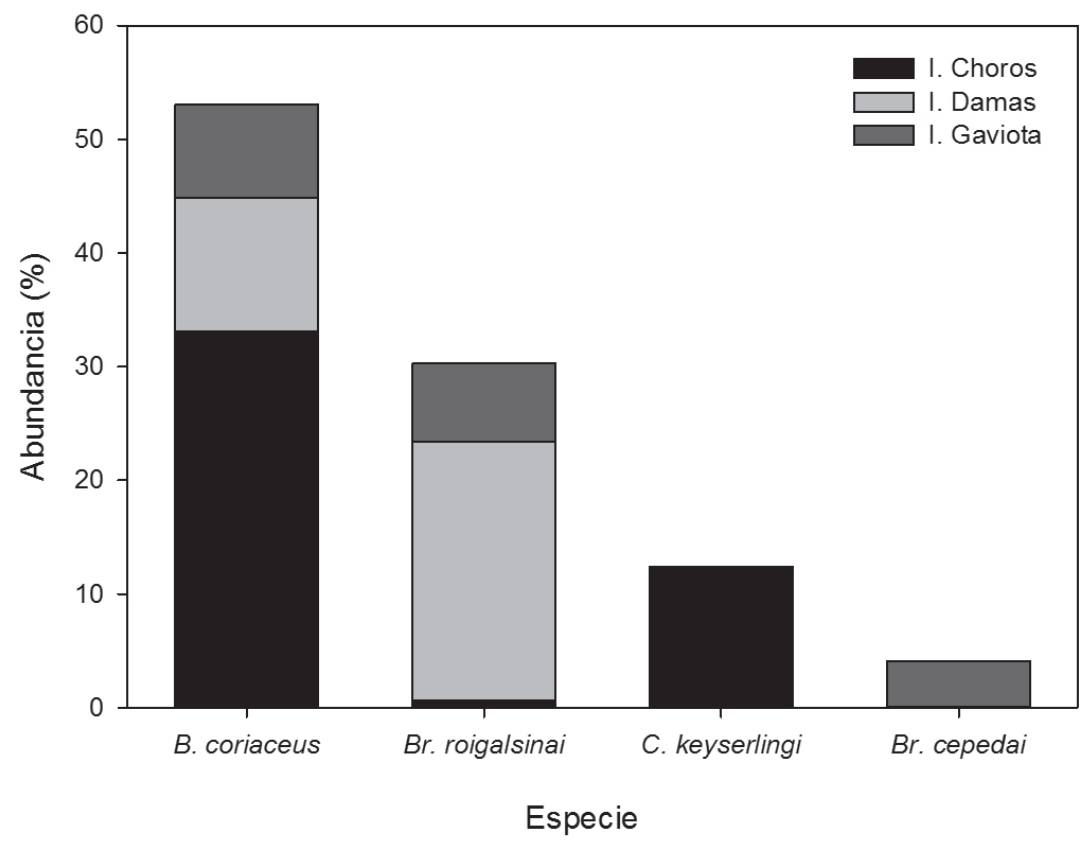

Figura 2. Abundancia de las especies registradas en cada una de las islas del Archipiélago de Los Choros.

FIGURE 2. Abundance of the species recorded in each island of Los Choros Archipelago.

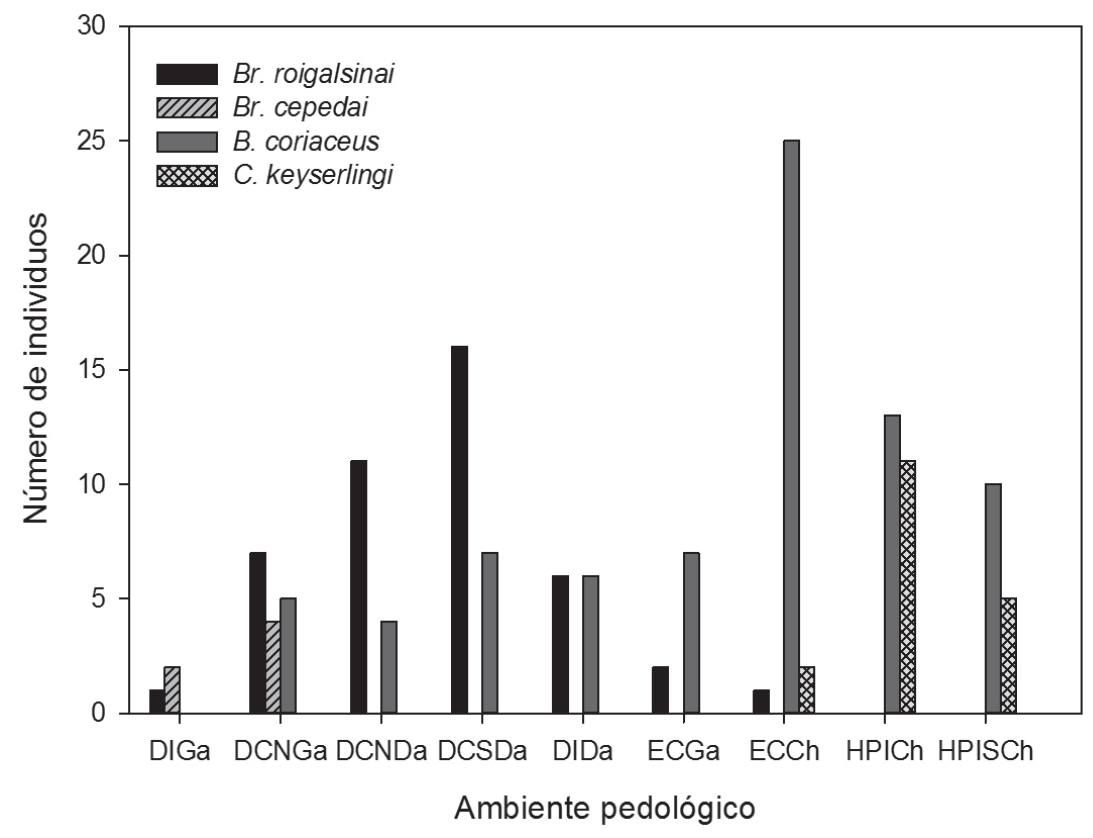

Figura 3. Presencia de las especies en cada uno de los ambientes pedológicos por isla.

Figure 3. Species presence in each of the pedological environments per island. 
El análisis temporal muestra que las especies B. coriaceus y $B r$.roigalsinai se registran en todas las estaciones estudiadas, mientras que $\mathrm{Br}$. cepedai, con una abundancia menor a las especies anteriores, sólo se registra en primavera y verano en la isla Gaviota (Fig. 4).

\section{SiMILITUD POR UNIDAD DE MUESTREO Y ESTACIONAL}

Mediante el análisis de similitud usando la matriz de BrayCurtis y la matriz de Jaccard, se encontró que no existen diferencias significativas en la composición de especies entre estación $(R=0,049 ; p=0,331)(R=0,20 ; p=0,97)$ (Fig. 5). Pero si habrían diferencias al considerar los ambientes pedológicos estudiados $(\mathrm{R}=0,523 ; \mathrm{p}=0,003)(\mathrm{R}=0,601 ; \mathrm{p}$ $=0,01)$ (Fig. 6).

El dendograma de estructuración espacial usando la matriz de Bray-Curtis muestran tres grupos con una similaridad mayor al 70 \%. Estos son: 1) el conformado mayoritariamente por ambientes de dunas costeras (i.e., DCNDa, DCSDa, DIDa, DCNGa, ECGa); 2) el grupo que reúne a los ambientes pedológicos pedregosos de la isla Choros y 3) dos unidades con una menor similitud que mezclan ambientes de duna interior y estepa costera (DIGa y ECCh) (Fig. 6).

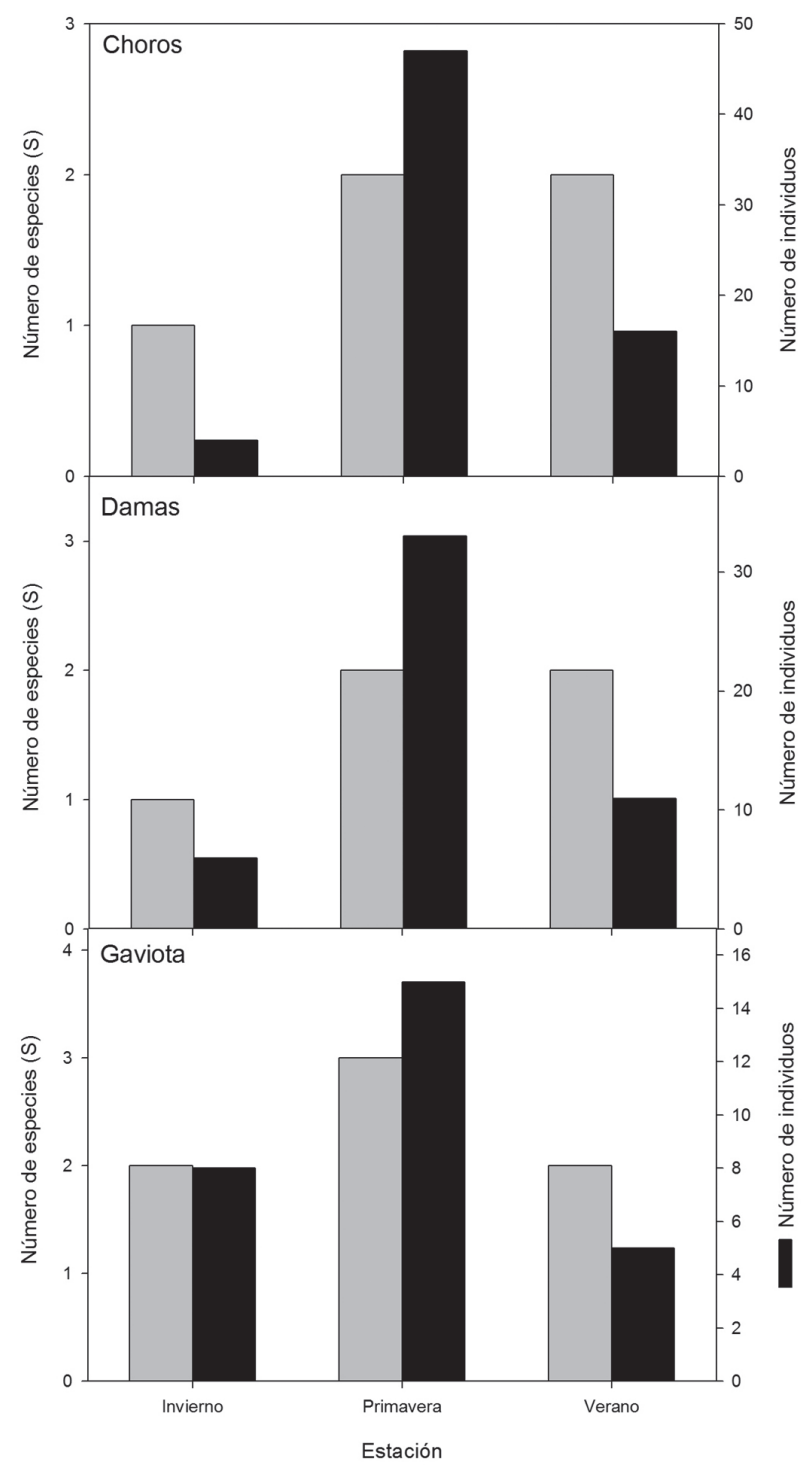

Figura 4. Riqueza y abundancia de especies en las distintas estaciones del año muestreadas en cada una de las islas del Archipiélago de Los Choros.

FiguRE 4. Abundance and species richness in the different seasons sampled along the year in each island of Los Choros Archipelago. 


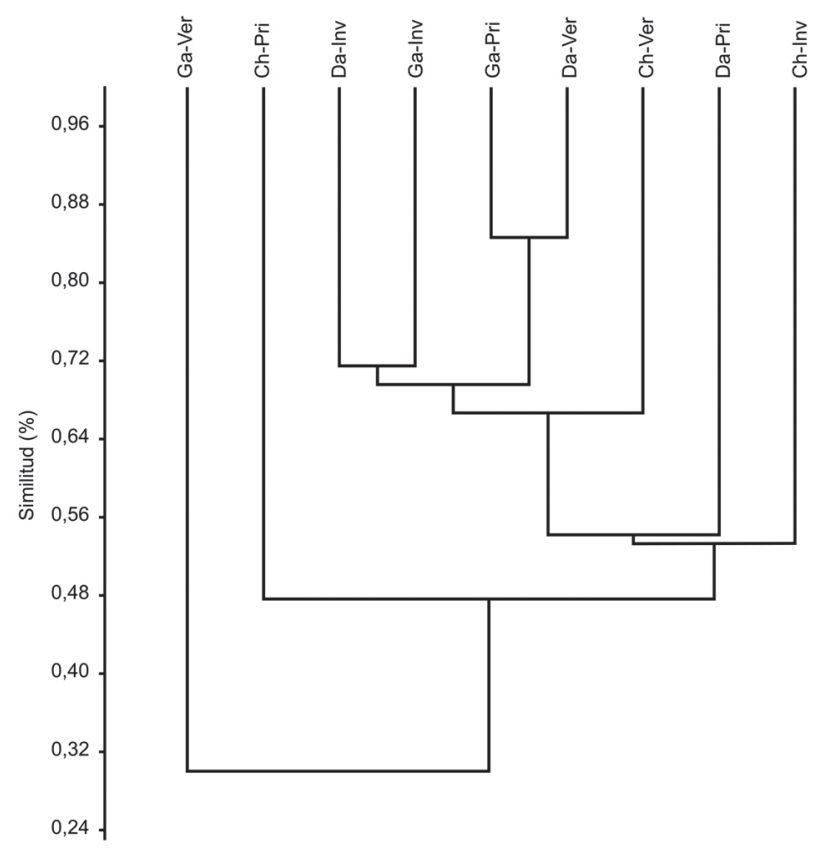

Figura 5. Estructuración estacional usando análisis de Cluster de la matriz de Bray-Curtis.

FIGURE 5. Seasonal structuring using the cluster analysis of Bray-Curtis matrix.

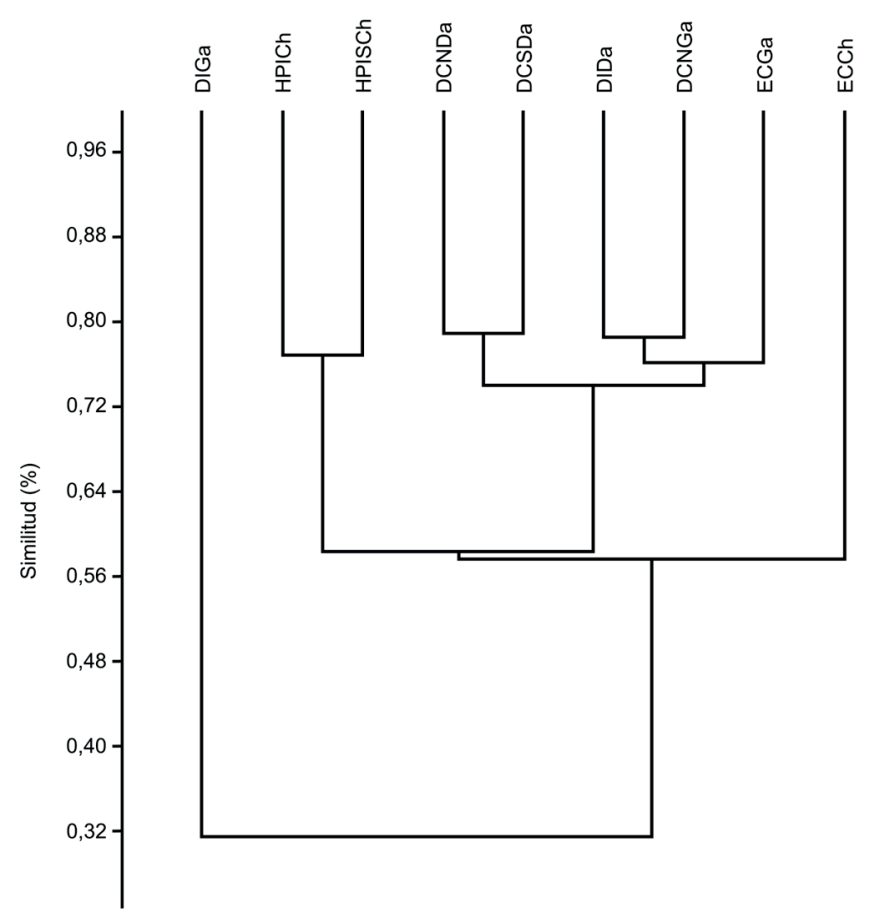

FiguRa 6. Estructuración por ambiente pedológico usando análisis de Cluster de la matriz de Bray-Curtis.

FIGURE 6. Structure per pedological environment using the cluster analysis of Bray-Curtis matrix. 


\section{DISCUSIÓN}

COMPOSICIÓN TAXONÓMICA DE LA ESCORPIOFAUNA

La escorpiofauna presente en el archipiélago de Los Choros se encuentra representada por dos familias, tres géneros y cuatro especies, las cuáles también han sido registradas en áreas continentales aledañas al archipiélago (Agusto et al. 2006). Las especies presentes en las islas seguramente forman parte de poblaciones, otrora continentales, que quedaron aisladas al separarse las islas del continente (Aguirre 1967). Debido a la poca vagilidad de las especies de Bothriuridae presentes en la zona, así como al tipo de ambiente y poblamiento, es improbable que estas hayan llegado vehiculizadas, natural o artificialmente. De las especies presentes en la zona continental cercana a la costa (Agusto et al. 2006), sólo Brachistosternus artigasi y Bothriurus dumayi no han sido colectadas en las islas; sin embargo la presencia de estas especies en esta latitud parece más bien marginal, por lo que no sorprende su ausencia en los ambientes insulares.

Se observa una tendencia unimodal de la abundancia y riqueza de especies con los valores menores en invierno y verano y el máximo en primavera, esto debido al ritmo biológico que presentan estas especies de escorpiones, ya que la mayoría de las actividades (i.e., reproducción, depredación) las realizan en los meses cálidos (Bradley 1982; Polis \& McCormick 1986). Esta diferencia de actividad estacional está determinada por una relación directa entre la temperatura de la superficie y la densidad de la abundancia (Fet 1980; Polis 1980; Warburg \& Polis 1990). Esto es más notorio para la especie Br. cepedai que sólo fue registrado en primavera y verano, y para C. keyserlingi, sólo registrado en ésta última estación. Colectas previas en el DCT indicaban que todas las especies conocidas de Bothriuridae del área poseen un ciclo de actividad estival (Ojanguren-Affilastro et al. 2007a, 2012); nuestros actuales resultados apoyan esto, y nos permiten inferir que el peak de actividad en superficie se da en primavera, cuando los machos buscan activamente hembras para reproducirse (Nime et al. 2013). Nuestros resultados nos permiten extrapolar también, una situación bastante similar al género Caraboctonus, del que hasta ahora casi no se tenían datos sobre su biología (Lourenço 1995).

Las características físicas del ambiente como la temperatura, precipitaciones y particularmente las propiedades del suelo son determinantes para comprender la distribución espacial de los escorpiones (Bradley 1986; Polis 1980, 1990; Prendini 2001). El análisis con la matriz de Jaccard mostró que existen diferencias en la abundancia entre los ambientes de las islas, pero no para las distintas fechas (estaciones). Esto último indica que las especies se encuentran presentes durante todo el año, pero con grandes fluctuaciones en su densidad en las diferentes estaciones, tal como fue notado anteriormente. A diferencia de Br. cepedai y de C. keyserlingi, las especies $B$. coriaceus y $\mathrm{Br}$. roigalsinai tienen actividad en todas las estaciones del año muestreadas, pero exhiben marcadas diferencias en la abundancia, la que tiende a ser mayor para ambas especies en primavera. Si bien la temperatura ambiental es un factor trascendental en la actividad de los escorpiones (Warburg \& Polis 1990), existe evidencia de que en la mayoría de las especies de escorpiones de zonas templadas y calidas, durante los meses fríos la actividad desciende, pero no cesa completamente (Polis 1980; Bradley 1982; Due \& Polis 1986; Ojanguren-Affilastro et al. 2011), $\mathrm{y}$ en este estudio se observaron evidencias de actividad invernal, aunque con poca abundancia para B. coriaceus y $\mathrm{Br}$. roigalsinai. Existen también especies que presentan su peak de actividad en invierno (Ojanguren-Affilastro et al. 2010, 2011), pero este no es el caso de ninguna de las especies colectadas en esta trabajo.

El hecho que existan diferencias relacionadas con la abundancia entre las distintas islas, reflejada por una diferencia de taxa que solo es relevante entre la isla Choros y Damas-Gaviota, pero no, entre Damas y Gaviota, reafirma las propuestas del efecto del micro-hábitat en la distribución y abundancia de los escorpiones (Polis 1990; McReynolds 2004; 2008), lo que implicaría que el sustrato y las formaciones vegetacionales presentes en cada hábitat influyen directamente en la presencia y niveles de abundancia de algunas especies de escorpiones (Polis 1980, 1990; Prendini 2001; McReynolds 2004, 2008). Esto es interesante ya que en el sistema insular estudiado, la cobertura vegetacional es baja y predominan los ambientes dunarios en las islas Damas y Gaviota, a diferencia de la isla Choros en la que es notorio el predominio de los ambientes pedregosos. Esta heterogeneidad de hábitat es mayor en las dos últimas islas, ya que también combinan la presencia de ambientes de estepa. La especie $B$. coriaceus habita en todos los ambientes (estepa, duna, pedregoso), mientras que $\mathrm{Br}$. roigalsinai es considerablemente más abundante en los ambientes dunarios, y su abundancia en ambientes de estepa es baja (7,0 \%). Para Br. cepedai sólo se registraron individuos en dunas, mientras que $C$. keyserlingi sólo fue colectada en ambientes pedregosos.

La separación existente entre las islas y el continente representa una barrera infranqueable para la mayoría de los artrópodos epígeos de desierto, ya que no poseen los medios para llegar vehiculizados a las mismas, por lo que son esperables fenómenos de especiación en ellas. De hecho el archipiélago de Los Choros, posee al menos un artrópodo epígeo endémico conocido: Gyriosomus granulipennis Pizarro-Araya \& Flores 2004 (Coleoptera: Tenebrionidae) (Pizarro-Araya \& Flores 2004; Alfaro et al. 2009; PizarroAraya et al. 2012a). 
Preliminarmente hemos detectado ligeras diferencias morfológicas entre los ejemplares continentales y los insulares de algunas de las especies colectadas; sin embargo, por el momento hemos decidido considerarlas como variaciones intra-específicas, aunque seguramente en el futuro, y con el aumento de material colectado, estas serán objeto de nuevos análisis.

\section{AGRADECIMIENTOS}

Agradecemos a Iván Benoit (Corporación Nacional Forestal, CONAF) y a CONAF Región de Coquimbo por la ayuda en los permisos y facilidades para trabajar en la Reserva Nacional Pingüino de Humboldt (Proyectos No 18/2011 y $\mathrm{N}^{\circ}$ 006/2014). A Claudio Canut de Bon (ex-académico del Departamento de Minas, Universidad de La Serena) por la entrega de información en relación a la geología y geomorfología del sitio de estudio. A Julio R. Gutiérrez por la lectura crítica del manuscrito (Departamento de Biología, Universidad de La Serena), a Luis Letelier (Centro de Investigaciones en Ecosistemas, Universidad Nacional Autónoma de México) por la asistencia en SIG, y a Marco Figueroa por la asistencia en las figuras (Departamento de Biología, Universidad de La Serena). El presente estudio fue financiado por los proyectos FPA 04-0152006 (CONAMA, Región de Coquimbo, Chile) y DIULSPR13121/VACDDI001 de la Universidad de La Serena, La Serena, Chile (JPA).

\section{BIBLIOGRAFÍA}

Aguirre, L. 1967. Geología de las islas Choros, Damas y de Punta Choros, Provincia de Coquimbo. Revista Minerales (Chile) 22: 73-83.

Agusto, P., Mattoni, C.I., Pizarro-Araya, J., Cepeda-Pizarro, J. \& López-Cortés, F. 2006. Comunidades de escorpiones (Arachnida: Scorpiones) del desierto costero transicional de Chile. Revista Chilena de Historia Natural 79: 407-421.

Alfaro, F.M., Pizarro-Araya, J. \& Flores, G.E. 2009. Epigean tenebrionids (Coleoptera: Tenebrionidae) from The Choros archipelago (Coquimbo Region, Chile). Entomological News 120: 125-130.

Arancio, G.A. \& JARA, P. 2007. Flora y Vegetación de la Reserva Nacional Pingüino de Humboldt. Ediciones Universidad de La Serena, La Serena, Chile. 71 pp.

Armesto, J.J., Vidiella, P.E. \& Gutiérrez, J.R. 1993. Plant communities of the free coastal desert of Chile: plant strategies in a fluctuating environment. Revista Chilena de Historia Natural 66: 271-282.

Benítez H.A., Pizarro-Araya, J., Bravi, R., Sanzana, M.J. \& Alfaro, F.M. 2014. Morphological variation on isolated populations of Praocis (Praocis) spinolai. Journal of Insect Science 14.11.

BradLEY, R.A. 1982. Digestion time and reemergence in the desert grassland scorpion Paruroctonus utahensis (Williams) (Scorpionida, Vaejovidae). Oecologia 55: 316-318.

BRADley, R.A. 1986. The relationship between population density of Paruroctonus utahensis (Scorpionida: Vaejovidae) and characteristics of its habitat. Journal of Arid Environments 11: $165-171$.

Bustamante, A.A., Scioscia, C.L. \& Casanueva, M.E. 2014. A new species of Admesturius Galiano, 1988 from north Chile (Araneae: Salticidae: Amycoida). Zootaxa 3774: 197-200.

Castro, C. \& Brignardello, L. 2005. Geomorfología aplicada a la ordenación territorial de litorales arenosos. Orientaciones para la protección, usos y aprovechamiento sustentables del sector de Los Choros, Comuna de la Higuera, IV Región. Revista de Geografía Norte Grande 33: 33-58.

Cavieres, L.A., Mihoc, M., Marticorena, A., Marticorena, C., Matthei, O. \& Squeo, F.A. 2001. Determinación de áreas prioritarias de conservación: análisis de parsimonia de endemismos (PAE) en la flora de la IV Región de Coquimbo. En: Libro rojo de la flora nativa y de los sitios prioritarios para su conservación: Región de Coquimbo (Eds. Squeo, F.A., Arancio, G. \& Gutiérrez, J.R.), 159-170. Ediciones Universidad de La Serena, Chile.

Cavieres, L.A., Arroyo, M.T.K., Posadas, P., Marticorena, C., Matthei, O., Rodríguez, R., Squeo, F.A. \& Arancio, G. 2002. Identification of priority areas for conservation in an arid zone: application of parsimony analysis of endemicity in the vascular flora of the Antofagasta region, northern Chile. Biodiversity and Conservation 11: 1303-1311.

Cepeda-Pizarro, J., Pizarro-Araya, J. \& Vásquez, H. 2005a. Composición y abundancia de artrópodos del Parque Nacional Llanos de Challe: impactos del ENOS de 1997 y efectos del hábitat pedológico. Revista Chilena de Historia Natural 78: 635-650.

Cepeda-Pizarro, J., Pizarro-Araya, J. \& VÁsquez, H. 2005 b. Variación en la abundancia de Arthropoda en un transecto latitudinal del desierto costero transicional de Chile, con énfasis en los tenebriónidos epígeos. Revista Chilena de Historia Natural 78: 651-663.

Elgueta, M. 2013. Geosphaeropterus, nuevo género de Tropiphorini (Coleoptera: Curculionidae) de Chile, con descripción de tres nuevas especies. Boletín del Museo Nacional de Historia Natural (Chile) 62: 203-217.

Di Castri, F. \& Hajek, E.R. 1976. Bioclimatología de Chile. Imprenta-Editorial de la Universidad Católica de Chile. Santiago, Chile. 128 pp.

Due, A.D. \& Polis, G.A. 1986. Trends in scorpion diversity along the Baja California peninsula. American Naturalist 128: 460-468.

Faith, D.P., Minchin, P.R. \& Belbin, L. 1987. Compositional dissimilarity as a robust measure of ecological distance. Vegetatio 69: 57-68.

FEt, V. 1980. Ecology of the scorpions (Arachnida, Scorpiones) of the southeastern Kara-Kum. Entomologicheskoe Obozrenie 59: 223-228.

Flores, G.E. \& Pizarro-Araya, J. 2012. Systematic revision of the South American genus Praocis Eschscholtz, 1829 (Coleoptera: Tenebrionidae). Part 1: Introduction and subgenus Praocis s. str. Zootaxa 3336: 1-35.

Gaston, K.J. 2000. Global patterns in biodiversity. Nature 405: 220-227. 
Hammer, Ø., Harper, D.A.T. \& Ryan, P.D. 2001. PAST: Palaeontological Statistics Software Package for Education and Data Analysis. Paleontología Electrónica 4: 1-9.

Klausmeyer, K.R. \& Shaw, M.R. 2009. Climate Change, Habitat Loss, Protected Areas and the Climate Adaptation Potential of Species in Mediterranean Ecosystems Worldwide. PLoS ONE 4: e6392.

Laborda, A., Ramirez, M.J. \& Pizarro-Araya, J. 2013. New species of the spider genera Aysenia and Aysenoides from Chile and Argentina: description and phylogenetic relationships (Araneae: Anyphaenidae, Amaurobioidinae). Zootaxa 3731: 133-152.

LOURENÇO, W.R. 1995. Considerations sur la morphologie, ecologie et biogeographie de Caraboctonus keyserlingi Pocock (Scorpiones, Iuridae). Boletín de la Sociedad de Biología de Concepción 66: 63-69.

Magurran, A.E. 1988. Ecological diversity and its measurement. Princeton University Press, New Jersey, USA. 179 pp.

Marticorena, C., Squeo, F.A., Arancio, G. \& Muñoz, M. 2001. Catálogo de la flora vascular de la IV Región de Coquimbo. En: Libro rojo de la flora nativa y de los sitios prioritarios para su conservación: Región de Coquimbo (Eds. Squeo, F.A., Arancio, G. \& Gutiérrez, J.R.), 105-142. Ediciones Universidad de La Serena, Chile.

Mattoni, C.I. 2007. The genus Bothriurus (Scorpiones, Bothriuridae) in Patagonia. Insect Systematics \& Evolution 38: 1-22.

Mattoni, C.I. \& Acosta, L.E. 2006. Systematics and distribution of three Bothriurus species (Scorpiones, Bothriuridae) from central and northern Chile. Studies on Neotropical Fauna and Environment 41: 235-250.

McReYnolds, C.N. 2004. Temporal patterns in microhabitat use for the scorpion Centruroides vittatus (Scorpiones, Buthidae). Euscorpius 17: 35-45.

McReynolds, C.N. 2008. Microhabitat preferences for the errant scorpion, Centruroides vittatus (Scorpiones, Buthidae). Journal of Arachnology 36: 557-564.

Nime, M.F., Casanoves, F., Vrech, D.E. \& Mattoni C.I. 2013. Relationship between environmental variables and surface activity of scorpions in the Arid Chaco ecoregion of Argentina. Invertebrate Biology 132: 145-155.

Novoa, R. \& Villaseca, S. 1989. (eds.) Mapa agroclimático de Chile. Instituto de Investigaciones Agropecuarias, Santiago, Chile. 221 pp.

OJANGUREN-AFFILASTRO,A.A. 2002. Nuevos aportes al conocimiento del género Brachistosternus en Chile, con la descripción de dos nuevas especies (Scorpiones, Bothriuridae). Boletín de la Sociedad de Biología de Concepción (Chile) 73: 3746.

Ojanguren-Affilastro, A.A. 2005. Notes on the genus Brachistosternus (Scorpiones, Bothriuridae) in Chile, with the description of two new species. Journal of Arachnology 33: $175-192$.

Ojanguren-Affilastro, A.A. \& Pizarro-Araya, J. 2014. Two new scorpion species from Paposo, in the Coastal desert of Taltal, Chile (Scorpiones, Bothriuridae, Brachistosternus). Zootaxa 3785: 400-418.

Ojanguren-Affilastro, A.A. \& Ramírez, M.J. 2009. Phylogenetic analysis of the scorpion genus Brachistosternus (Arachnida, Scorpiones, Bothriuridae). Zoologica Scripta
38: 183-198.

Ojanguren-Affilastro, A.A., Pizarro-Araya, J. \& Prendini, L. 2011. New data on Chilean Urophonius (Scorpiones, Bothriuridae). American Museum Novitates 3725: 1-44.

Ojanguren-Affilastro, A.A., Agusto, P., Pizarro-Araya, J. \& Mattoni, C.I. 2007a. Two new scorpion species of genus Brachistosternus (Scorpiones: Bothriuridae) from northern Chile. Zootaxa 1623: 55-68.

Ojanguren-Affilastro, A.A., Mattoni, C.I. \& Prendini, L. 2007b. The genus Brachistosternus (Scorpiones: Bothriuridae) in Chile, with descriptions of two new species. American Museum Novitates 3564: 1-44.

Ojanguren-Affilastro, A.A., OchoA, J.A., Mattoni, C.I. \& Prendini, L. 2010. Systematic revision of the granulatus group of Urophonius Pocock, 1893 (Scorpiones, Bothriuridae), with description of a new species from central Chile. American Museum Novitates 3695: 1-40.

Ojanguren-Affilastro, A.A., Mattoni, C.I., OchoA, J.A. \& Prendini, L. 2012. Rumikiru, n. gen. (Scorpiones: Bothriuridae), a new scorpion genus from the Atacama desert. American Museum Novitates 3731: 1-43.

Pizarro-Araya, J. \& Flores, G.E. 2004. Two new species of Gyriosomus Guérin-Méneville from Chilean coastal desert (Coleoptera: Tenebrionidae: Nycteliini). Journal of the New York Entomological Society 112: 121-126.

Pizarro-Araya, J. \& Jerez, V. 2004. Distribución geográfica del género Gyriosomus Guérin-Méneville, 1834 (Coleoptera: Tenebrionidae): una aproximación biogeográfica. Revista Chilena de Historia Natural 77: 491-500.

Pizarro-Araya, J., Vergara, O.E. \& Flores, G.E. 2012a. Gyriosomus granulipennis Pizarro-Araya y Flores 2004 (Coleoptera: Tenebrionidae) un caso extremo a conservar. Revista Chilena de Historia Natural 85: 345-349.

Pizarro-Araya, J., Alfaro, F.M., Castillo, J.P., OjangurenAffilastro, A.A., Agusto, P. \& Cepeda-Pizarro, J. 2012 b. Assemblage of arthropods in the Quebrada del Morel private protected area (Atacama Region, Chile). Pan Pacific Entomologist 88: 1-14.

Polis, G.A. 1980. Seasonal patterns and age-specific variation in the surface activity of a population of desert scorpions in relation to environmental factors. Journal of Animal Ecology 49: 1-18.

Polis, G.A. 1990. Ecology. En: The Biology of Scorpions (Ed. Polis, G.A.), 247-293. Stanford. Stanford University Press. Stanford, California, USA.

Polis, G.A. 1993. Scorpions as model vehicles to advance theories of population and community ecology: The role of scorpions in desert communities. Memoirs of the Queensland Museum 33: 401-410.

Polis, G.A. \& Farley, R.D. 1980. Population Biology of a Desert Scorpion: Survivorship, Microhabitat, and the Evolution of Life History Strategy. Ecology 61: 620-629.

Polis, G.A. \& McCormick, S.J. 1986. Patterns of resource use and age structure among species of desert scorpion. Journal of Animal Ecology 55: 59-73.

Prendini, L. 2001. Substratum specialization and speciation in southern African scorpions: the effect hypothesis revisited. En: Scorpions 2001. In Memoriam Gary A. Polis (Eds. Fet, V. \& Selden, P.A.), 113-138. British Arachnological Society, Burnham Beeches, Bucks, UK. 
StahnKe, H.L. 1972. UV light, a useful field tool. BioScience 22: 604-607.

Valdivia, D.E., Pizarro-Araya, J., Cepeda-Pizarro, J. \& OJANGUREn-Affilastro, A.A. 2008. Diversidad taxonómica y denso-actividad de solífugos (Arachnida: Solifugae) asociados a un ecosistema desértico costero del centro norte de Chile. Revista de la Sociedad Entomológica Argentina 67: 1-10.

Valdivia, D.E., Pizarro-Araya, J., Briones, R., OjangurenAffilastro, A.A. \& Cepeda-Pizarro, J. 2011. Taxonomical diversity and abundance of solpugids (Arachnida: Solifugae) in coastal ecotopes of north-central Chile.
Revista Mexicana de Biodiversidad 82: 1234-1242.

Vidiella, P.E., Armesto, J.J. \& Gutiérrez, J.R. 1999. Vegetation changes and sequential flowering after rain in the southern Atacama Desert. Journal of Arid Environments 43: 449458.

Warburg, M.R. \& Polis, G.A. 1990. Behavioral responses, rhythms, and activity patterns. En: Polis GA (ed) The Biology of Scorpions. Stanford University Press. Stanford, California, USA. 587 pp.

Williams, S.C. 1987. Scorpion Bionomics. Annual Review of Entomology 32: 275-295.

Recibido: 30.07 .13

Aceptado: 08.04.14 